\title{
Caracterización del diseño de la suspensión inclinable para vehículos de movilidad personal
}

\author{
Fernando E. Maldonado-Páez ${ }^{1,2 \star}$, Edilberto A. Llanes-Cedeño1, Gonzalo E. Guerrón-López ${ }^{1,3}$ y Juan C. Rocha-Hoyos ${ }^{1,4}$ \\ (1) Univ. Particular Internacional SEK, Fac. de Arquitectura e Ingenierías, Casilla 17-03-02, Quito, Ecuador. \\ (correo-e: antonio.llanes@uisek.edu.ec, gonzalo.guerron@uisek.edu.ec) \\ (2) Inst. Sup. Tecn. Francisco de Orellana, Puyo - Ecuador. (correo-e: fernando.maldonado@itsfo.edu.ec) \\ (3) Univ. Tecn. Equinoccial, Facultad de Ciencias de la Ingeniería e Industrias, Quito, Ecuador. \\ (correo-e: gonzalo.guerron@ute.edu.ec) \\ (4) Esc. Sup. Politécnica del Chimborazo, Facultad de Mecánica, Riobamba, Ecuador. \\ (correo-e: juan.rocha@espoch.edu.ec)
}

* Autor a quien debe ser dirigida la correspondencia.

Recibido Sep. 9, 2019; Aceptado Nov. 11, 2019; Versión final Ene. 9, 2020, Publicado Jun. 2020

\begin{abstract}
Resumen
El presente artículo estudia el proceso de diseño e implementación de las suspensiones inclinables en coches angostos. Los coches angostos son un nuevo tipo de automóvil que podría llegar a solucionar los problemas de congestión, la contaminación ambiental y falta de espacios de estacionamiento en las urbes. Sin embargo, sus dimensiones no solo acarrean beneficios, ya que presentan inconvenientes en la implementación de suspensiones activas inclinables. Para estudiar la implementación de las suspensiones activas inclinables se realiza una investigación exploratoria, empleando estudios acerca del tema y aplicando la Declaración PRISMA. Se realizó un análisis explicativo entre varios modelos construidos y propuesto de suspensión, con respecto al diseño del mecanismo y su sistema de control, estableciéndose un proceso de diseño, implementación, control y simulación. Al evaluar los métodos tradicionales y programas de simulación se concluye que estos últimos ayudan a realizar la comprobación del modelo matemático.
\end{abstract}

Palabras clave: suspensión inclinable; impacto ambiental; vehículos unipersonales; vehículos angostos; diseño de suspensión

\section{Characterization of the tilt suspension design for personal mobility vehicles}

\begin{abstract}
This article studies the process of design and implementation of tiltable suspensions in narrow cars. Narrow cars are a new type of vehicle that could solve the problems of congestion, environmental pollution, and lack of parking spaces in cities. However, the size of narrow cars does not only bring benefits, but it also carries challenges on the implementation of tiltable suspensions. To study the implementation of tiltable suspensions on narrow cars, is performed an exploratory investigation using data from previews studies on the subject and is applied the PRISMA Declaration. Our analysis compared previously built and proposed tiltable suspension models, including their respective mechanism designs control systems. We established a design, an implementation, and a control and simulation process for tiltable suspensions. Comparison between traditional methods and simulation software showed that simulation software can assist on testing the mathematical model.
\end{abstract}

Keywords: active suspension; environmental impact; narrow tilting vehicle; tilting vehicles; suspension design 


\section{INTRODUCCIÓN}

La necesidad de cambiar el diseño de los vehículos de transporte convencional, a modelos más eficientes para la movilidad urbana, ha obligado a los fabricantes a extender a los usuarios una nueva tendencia con respecto al diseño. Según Claveau et al., (2014) y Tan et al., (2016), una nueva generación de coches está siendo estudiada la cual será práctica y eficiente en relación con los problemas de tráfico y falta de espacios de estacionamiento en las áreas urbanas. Estos vehículos son angostos y pequeños, por lo tanto, el ahorro de combustible y su tamaño reducido a la mitad de los convencionales (menor a $1 \mathrm{~m}$ ) serían una ventaja al momento de movilizarse (Rocha et al., 2018).

A futuro, el transporte unipersonal se plantea como una alternativa eficiente de movilidad, que pretende resolver varios problemas con respecto al tránsito en los centros poblados. Berote et al., (2015) y Antony et al., (2016), menciona que, los vehículos con ancho de vía angosta pueden ser construidos con una significativa reducción de peso y su área frontal disminuida, respecto a los ordinarios, además de ocupar menor huella en la vía. Furuichi et al., (2013) y Amodio et al., (2017) en sus trabajos, añaden que, su desarrollo y aplicación a la transportación diaria, aportarían significativamente a la solución de los problemas mencionados, debido a que estos vehículos necesitan menos espacio para aparcar, menor espacio de vía y disminución de la contaminación (Rocha et al., 2019).

De esta manera surgen los vehículos angostos inclinable (Narrow Tilting Vehicles). Los NTV's son la fusión entre un automóvil y una motocicleta, generando una gran expectativa para la nueva generación de transporte urbano, considerando sus dimensiones prácticas y su menor consumo de energía. Berote et al. (2015) indica que, con el fin de que sea seguro y confortable como un coche convencional, debe ser alto y cerrado, pero debido a que por naturaleza una estructura alta y angosta tiende al vuelco o derrape, es necesario inclinarlo hacia el interior de la curva, esto con la finalidad de compensar el momento generado por la fuerza lateral, en adición Claveau et al., (2014) y Kim et al., (2014) menciona que, a diferencia del caso de una motocicleta, en donde el conductor inclina por sí mismo, la inclinación del NTV debe ser automática. Dos sistemas de control están disponibles, el control directo de inclinación y el control de dirección de inclinación, la acción combinada de estos dos sistemas sería la clave para mejorar el comportamiento dinámico (Guamán et al., 2019). El presente artículo realiza un estudio del proceso de análisis dinámico y las herramientas empleadas para el proceso de validación del control y simulación de un sistema de suspensión inclinable para vehículos de transporte personal (NTV).

\section{METODOLOGÍA}

Mediante una revisión sistemática se realiza la revisión de documentos y publicaciones referentes al tema. La declaración PRISMA (Preferred Reporting Items for Systematic reviews and Meta-Analyses) de Dios et al.,, es utilizada en este documento para la búsqueda, identificación, selección y evaluación objetiva de estos documentos, con el objetivo de extraer e interpretar los resultados obtenidos de los estudios concluidos. De esta manera las preguntas que se pretende responder son: 1) ¿Cuáles son las características geométricas, cinemáticas y dinámicas de una suspensión Tilting para vehículos de transporte personal?; 2) ¿Cuál es el proceso de diseño, control e implementación de una suspensión tilting para un vehículo de transporte personal?; y 3) ¿Cómo las suspensiones tipo tilting mejoran el comportamiento dinámico del vehículo?.

El estudio se realiza empleando artículos y publicaciones de las siguientes bases de datos bibliográficos: ASME, IEEE, Latindex, Reserch Gate, SAE, SAGE, Scielo, Science Direct, Scopus, Taylor \& Francis y otros (Repositorios universitarios, archivos de patentes). En las cuales se aplicaron como criterios de búsqueda las siguientes palabras clave y expresiones: suspension tilting, suspension active, suspension leaning, suspensión basculante, suspensión inclinable, narrow tilting vehicle, urban vehicles, tilting vehicles, suspension mechanism design, variable wheelbase, control systems, lógica difusa, inteligencia artificial, de los cuales se obtuvo artículos de investigación, artículos de revisión, enciclopedias, capítulos de libros, editoriales, noticias, patentes, artículos informativos, minireseñas. Los resultados obtenidos fueron procesados para la aplicación de la metodología PRISMA, y se presentan en la Tabla 1. La búsqueda realizada arroja como resultados un total de 193 documentos. El proceso de selección de la documentación e información referente al tema propuestos se describe en la figura 1. Los registros obtenidos finalmente de las 9 bases de datos citadas finalmente son 43, las cuales serán utilizadas para el desarrollo de la presente investigación.

\section{RESULTADOS, ANALISIS Y DISCUSIÓN}

Con el objetivo de proporcionar al diseñador una guía para el entendimiento y desarrollo de la tecnología, en busca de la eficiencia en el diseño y construcción de sistemas respecto a la presente tipología de vehículo (Valderrama, 2005; Vega et al., 2018). El primer punto es el Análisis de la geometría: Se establece los 
parámetros de particulares mediante la comparación de algunos modelos, entre sus mecanismos y dimensiones útiles en la construcción del NTV con el fin de establecer sus características; Modelado matemático: Se presentan algunas propuestas para el estudio matemático del modelo del NTV como son: el péndulo invertido, fórmula mágica de Pacejka y modelos multicuerpo, estos métodos de análisis, permitirán predecir el comportamiento del vehículo con respecto a los esfuerzos estáticos y dinámicos aplicados (Cardona et al., 2019); Diseño del sistema de control: Describe las técnicas utilizadas con frecuencia para el control de actuadores del NTV, parte importante en el desarrollo de este tipo de vehículos, ya que por medio del control se pretende mitigar los efectos de la aceleración lateral sobre la seguridad, confort y percepción del vehículo hacia el conductor; Simulación: Resulta costoso fabricar un vehículo que disponga de un sinnúmero de sensores para poder medir las magnitudes solicitadas, además de riesgoso. La tecnología actual permite realizar simulaciones aproximadas por medio de asistencia computacional, en este sentido se analiza algunas opciones para la realización de este proceso.

Tabla 1: Procesamiento de datos

\begin{tabular}{|c|c|c|c|c|}
\hline Palabras clave & Base de Datos & Encontrados & Seleccionados & Porcentaje \\
\hline \multirow{10}{*}{ 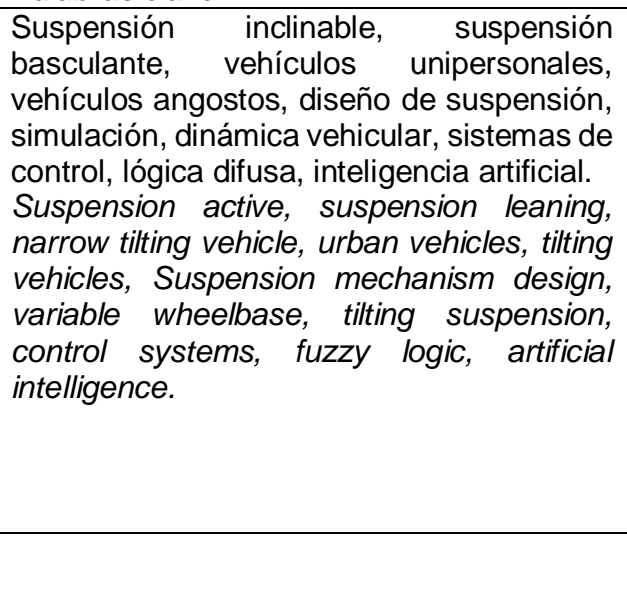 } & ASME & 4 & 1 & $2.2 \%$ \\
\hline & IEEE & 20 & 7 & $15.2 \%$ \\
\hline & Reserch Gate & 6 & 2 & $4.3 \%$ \\
\hline & SAE & 22 & 2 & $4.3 \%$ \\
\hline & SAGE & 28 & 5 & $10.9 \%$ \\
\hline & Scielo & 7 & 7 & $15.2 \%$ \\
\hline & Science Direct & 17 & 6 & $13.0 \%$ \\
\hline & Taylor \& Francis & 9 & 2 & $4.3 \%$ \\
\hline & Otros & 80 & 14 & $30.4 \%$ \\
\hline & Total & 193 & 46 & $100.0 \%$ \\
\hline
\end{tabular}

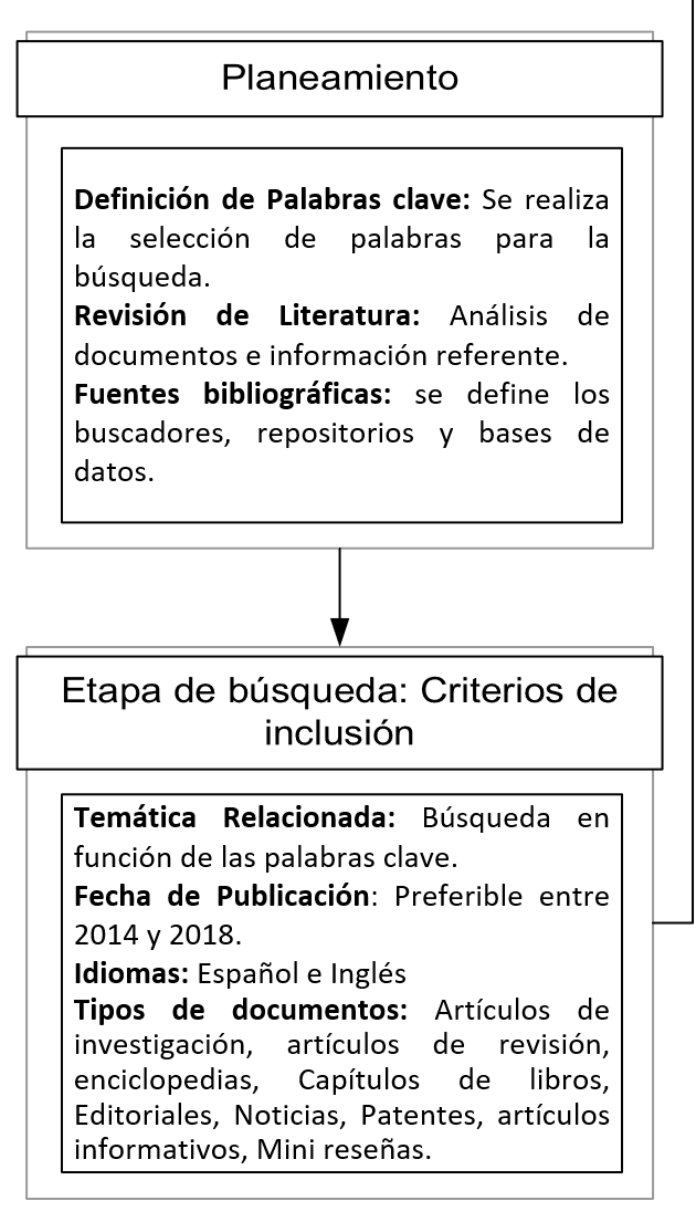

Etapa de búsqueda: Criterios de Exclusión

Se descartan los documentos:

-Que a pesar de contener las palabras claves, su información no corresponde al tema de investigación.

-Que son informativos y no técnicos.

-Que no son académicos, como los artículos de opinión.

Bases de datos utilizadas: 9

Documentos encontrados: 193

Documentos Seleccionados: 43

Fig 1. Proceso de selección de registros metodología PRISMA 


\section{Geometría del vehículo inclinable angosto (NTV)}

De acuerdo con Mourad et al., (2014), los NTV's considerando su geometría tienen aproximadamente $2.5 \mathrm{~m}$ de largo, $1 \mathrm{~m}$ de ancho y $1.5 \mathrm{~m}$ de alto, estos vehículos se caracterizan por tener un centro de gravedad elevado. Dong et al., (2014) y Furuichi et al., (2013) señalan que, algunos proyectos de tres y de cuatro ruedas han sido propuestos por algunas compañías. El vehículo Ford Gyron mostrado en la figura $2 a$ fue uno de los primeros, mientras que General Motors desarrollo la "Lean machine" (figura 2b) con un sistema de inclinación manual controlado por el conductor. Recientemente la empresa Brink Dynamics desarrollo el Carver (figura 2c), un vehículo de tres ruedas con carrocería inclinable, pero un tren de potencia trasero no inclinable.
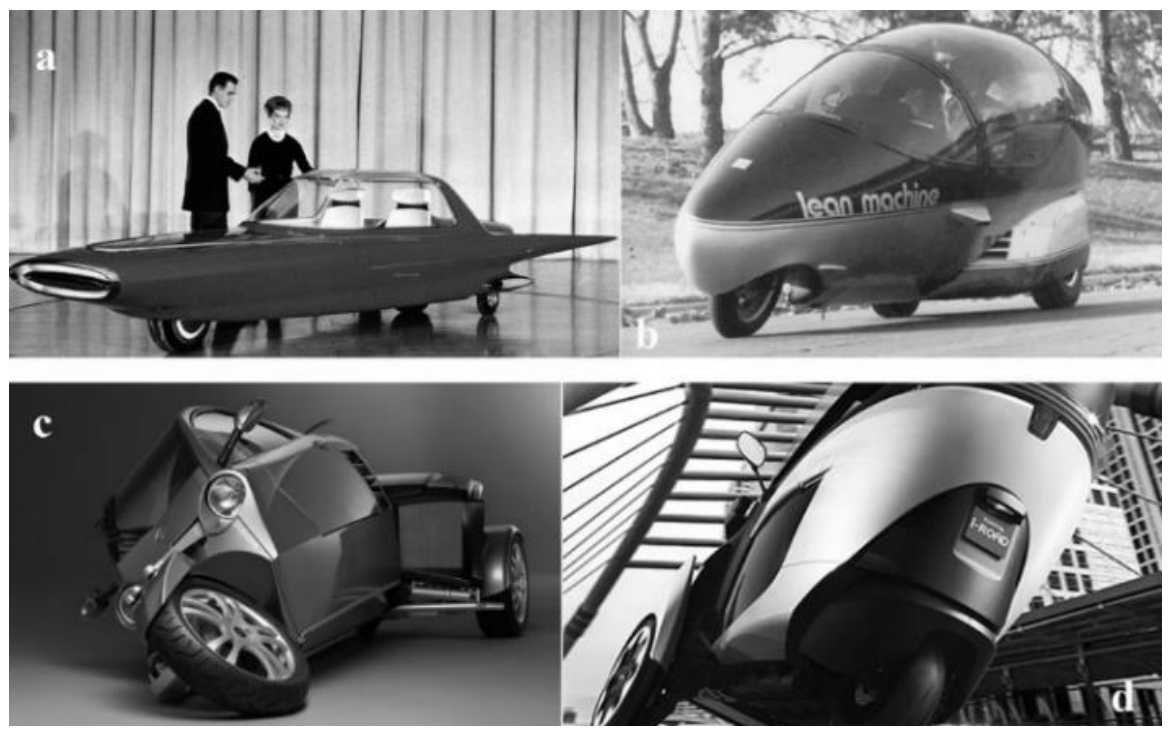

Fig 2. (a) Gyron, (b) Lean machine, (c) Carver, (d) Toyota I-road.

Este tipo de vehículos tienen varios problemas técnicos. Al ser vehículos de tres ruedas son más susceptibles al viraje y al derrape que los vehículos de cuatro ruedas. En la actualidad, se plantea el uso de tecnología "drive by wire" (control electrónico por cable), para permitir un control total sobre los sistemas del vehículo. El Toyota I-Road (figura 2d) y el Smera son una muestra del avance tecnológico que los NTV's han experimentado; este prototipo posee un novedoso sistema de suspensión activa llamada Inclinación activa (active lean). El sistema está compuesto por mecanismos controlados electrónicamente: motores eléctricos, actuadores hidráulicos, servomotores, etc.

Modelo 1: El Clever, consiste en una cabina basculante con el espacio para el conductor y un pasajero, y un módulo trasero de propulsión no basculante, el cual contiene el motor y los accesorios. Un sistema de control en lazo cerrado toma las señales de velocidad del vehículo y el ángulo de inclinación como entradas para controlar dos actuadores hidráulicos los cuales inclinan la cabina respecto al módulo trasero, en la figura 3 se muestra un esquema del vehículo.

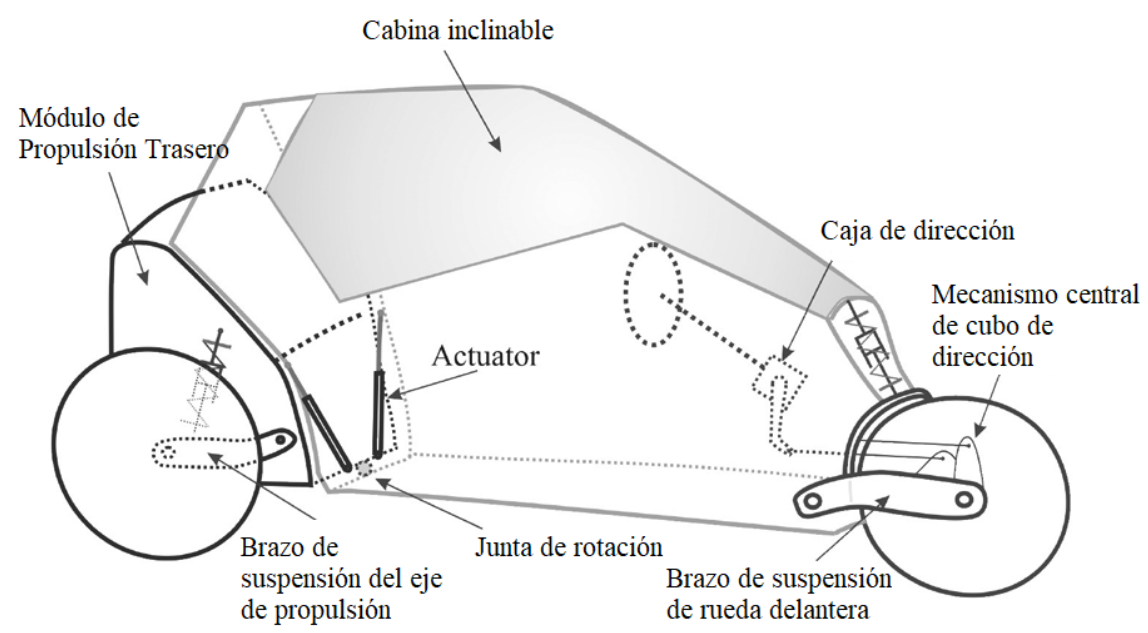

Fig 3. Representación esquemática del Clever (Adaptada de Berote et al., 2015) 
La geometría de la suspensión y la caracterización de la rigidez y amortiguación son de vital importancia para determinar el comportamiento dinámico del vehículo. La suspensión trasera en particular tiene la función de reaccionar a las fuerzas que producen los actuadores y mantener la estabilidad de la plataforma para el sistema de inclinación. El módulo trasero usa un brazo basculante con eje rígido provisto de amortiguadores, y configurada para brindar un comportamiento casi lineal entre el movimiento vertical de la rueda y el movimiento de compresión del amortiguador, posicionando este último de manera tangencial al arco del brazo basculante. Además, se muestra el mecanismo de cubo central de dirección, la rueda frontal está unida al chasis por dos brazos basculantes paralelos y una sola unidad de amortiguación (resorte - amortiguador), como menciona Berote et al., (2015), tal como se resume en la tabla 2.

Tabla 2: Características del Clever (Adaptada de Berote et al., 2015)

\begin{tabular}{|c|c|}
\hline \multicolumn{2}{|l|}{ General: } \\
\hline Tipo: & Tres ruedas controladas por inclinación directa \\
\hline Construcción: & Marco de aluminio con paneles de plástico reforzado con vidrio \\
\hline Control de conducción: & Volante y pedales (sistema similar a un coche) \\
\hline Ocupantes: & $1+1$ asientos en tándem \\
\hline \multicolumn{2}{|l|}{ Dimensiones y masas } \\
\hline Longitud: & $3 \mathrm{~m}$ \\
\hline Ancho: & $1 \mathrm{~m}$ \\
\hline Altura: & $1.35 \mathrm{~m}$ \\
\hline Distancia entre ruedas: & $0.84 \mathrm{~m}$ \\
\hline Batalla: & $2.4 \mathrm{~m}$ \\
\hline Masa del vehículo: & $350 \mathrm{~kg}$ \\
\hline \multicolumn{2}{|l|}{ Neumáticos y suspensión } \\
\hline Neumático delantero: & 120/R17 Motocicleta \\
\hline Neumático trasero: & 160/50R18 Personalizado \\
\hline Suspensión delantera: & Brazo oscilante con amortiguador ajustable y dirección central del cubo \\
\hline Suspensión trasera: & Doble brazo de arrastre con estabilizador de vuelco y amortiguadores ajustables \\
\hline Inclinación: & $17^{\circ}$ \\
\hline Ancho de vía: & $91 \mathrm{~mm}$ \\
\hline Relación de dirección: & $12: 1$ \\
\hline \multicolumn{2}{|l|}{ Motor y transmisión } \\
\hline Motor: & $230 \mathrm{~cm} 3 \mathrm{BMW}$ C1 scooter (13kW at $9000 \mathrm{r} / \mathrm{min})$ \\
\hline Transmisión: & $\begin{array}{l}\text { Transmisión continuamente variable con diferencial; concéntrico dentado último } \\
\text { accionamiento de la correa }\end{array}$ \\
\hline \multicolumn{2}{|l|}{ Rendimiento } \\
\hline $0-60$ km/h: & $7 \mathrm{~s}$ \\
\hline Velocidad máxima: & $100 \mathrm{~km} / \mathrm{h}$ \\
\hline Radio de curva: & $8-9 m$ \\
\hline \multicolumn{2}{|l|}{ Seguridad } \\
\hline Pasiva: & $\begin{array}{l}\text { Jaula de seguridad absorbente de energía con un airbag del conductor y un cinturón de } \\
\text { seguridad de tres puntos }\end{array}$ \\
\hline
\end{tabular}

Modelo 2: Furuichi, la configuración de este modelo es semejante que el Clever, de dos ruedas traseras montadas en un tren de impulsión y una frontal. El modelo presentado tiene ruedas posteriores que se inclinan y una rueda frontal que se inclina con el chasis del vehículo por medio de un par de torsión que genera un motor eléctrico y un sistema de control electrónico como se aprecia en la figura 4.

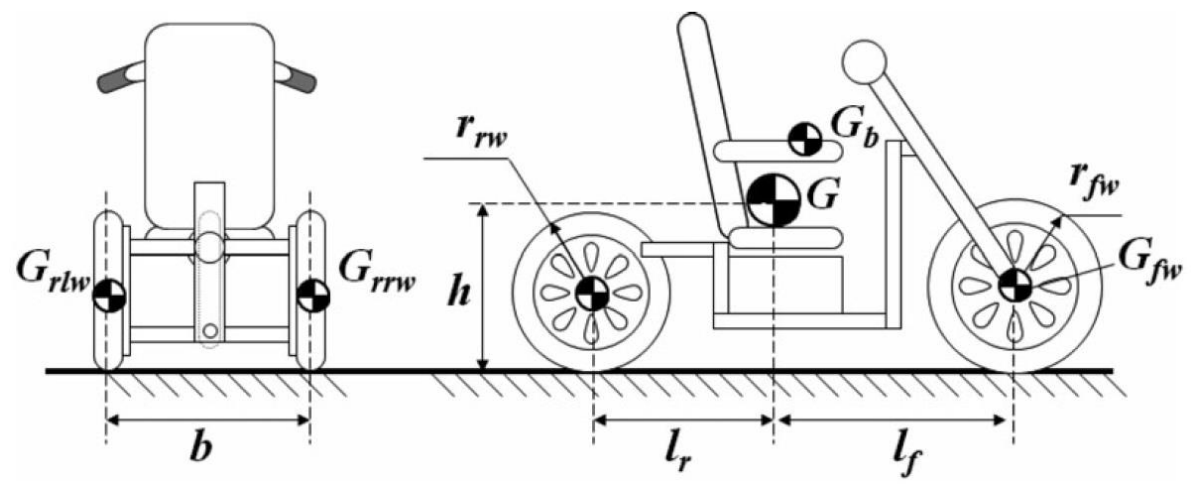

Fig 4. NTV Conceptual vista trasera y lateral (Adaptada de Furuichi et al., 2013) 
Modelo 3:SMERA, las dimensiones del Smera son $2.5 \mathrm{~m}$ de largo, $0.96 \mathrm{~m}$ de ancho, y de alto $1.45 \mathrm{~m}$. Estas dimensiones permiten al vehículo moverse de manera ágil en las áreas urbanas. Está equipado con un Sistema de inclinación motorizado que permite inclinar de manera simultánea las cuatro ruedas del vehículo con respecto al chasis, visto en la figura 5 . En el diagrama de la figura $6 \mathrm{~b}$ se muestran varios eslabones unidos mediante juntas (círculos), cada cuerpo está asociado con un color ilustrados físicamente en el diagrama a la izquierda, figura 6a. El tren trasero está compuesto de una pieza mecánica llamada Lira, movida por un motor cuyo estator está fijado a la carrocería del vehículo, la parte superior está conectada por medio de dos juntas esféricas a los dos conjuntos resorte amortiguador, cuya parte inferior se conecta a su correspondiente brazo de suspensión que se conectan por el otro extremo al chasis por medio de una junta revoluta.

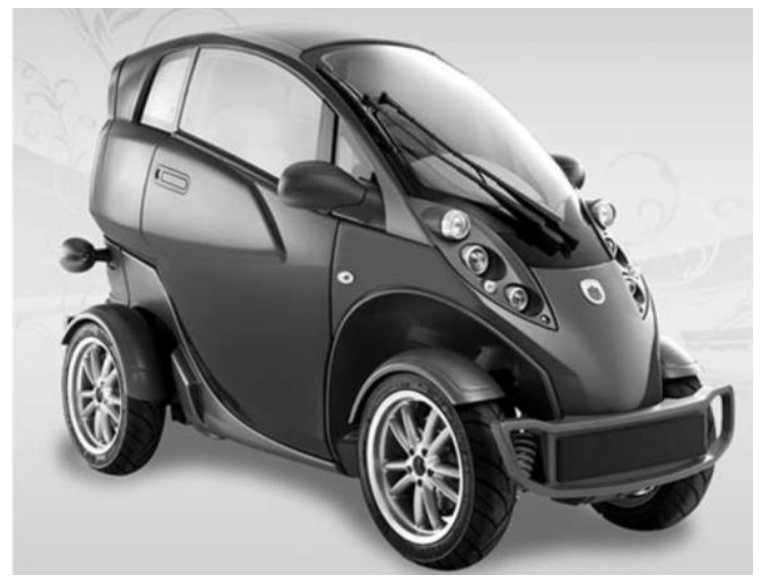

Fig 5. Smera (Adaptada de Maakaroun et al., 2013)

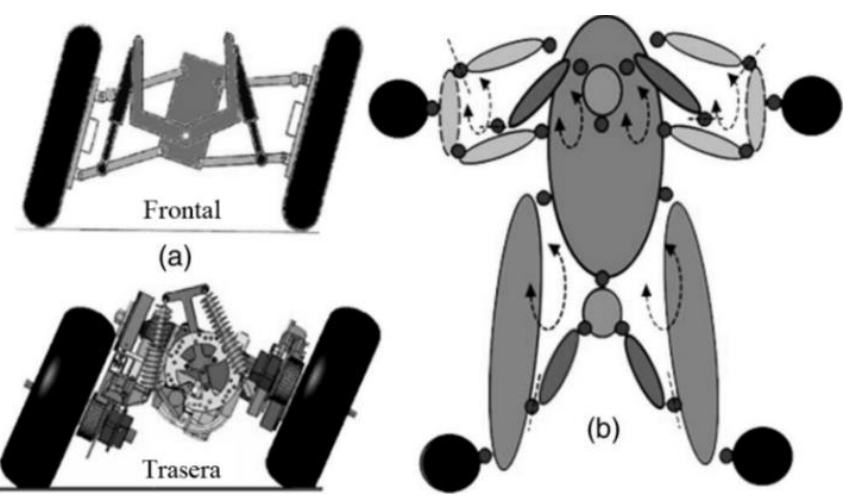

Fig 6. Diagrama de componentes del Smera (Adaptada de Maakaroun et al., 2013)

\section{Modelado matemático}

La metodología empleada consiste en determinar el comportamiento del vehículo en diferentes condiciones. Existen varios estudios realizado en donde se aplican una variedad de métodos como la fórmula mágica de Pacejka et al., (1992) la cual se emplea en varios estudios como los de: Maakaroun et al., (2013); Kim et al., (2014); Chatterjee et al., (2015); Schommer et al., (2015); Ma et al., (2017); Németh et al., (2017); Syed et al., (2016); Nguyen et al., (2018); y Onsy et al., (2018). El método de péndulo invertido utilizado por Dong et al., (2014); Furuichi et al., (2013); y Claveau et al., (2014). Análisis Multicuerpo empleado por Corno et al., (2015); Tognolli et al., (2015), así como, Tan et al., (2016). El Principio de D'Alembert es utilizado en las investigaciones de Nagarkar et al., (2018); Yao et al., (2018). Los cuales permiten obtener ecuaciones que describen el movimiento del vehículo tales como, la fuerza de frenado, fuerza lateral y par autoalineante en términos de deslizamiento longitudinal y ángulo de deriva. Los principios mencionados generalmente proporcionan ecuaciones diferenciales o integrales, las cuales es necesario procesarlas para generar ecuaciones de transferencia, que serán procesadas en los sistemas de control, estas deben estar linealizadas expresadas como ecuaciones algebraicas, que determinadas por medio de métodos numéricos, permitan a los controladores procesar la información. Las técnicas empleadas con este fin también varían y pueden ser: Lagrange, Newton- Euler, Linearización Jacobiana, o el método de Runge Kutta.

\section{Método fórmula mágica: Ecuaciones de movimiento}

Hans B. Pacejka fue un experto en dinámica de vehículos, especializado en la dinámica de la rueda. De manera empírica sus fórmulas matemáticas permiten determinar el comportamiento de las fuerzas y 
momentos que actúan entre la rueda del vehículo y la carretera, permitiendo determinar de manera precisa, el comportamiento del neumático en estado estable y de manera general el comportamiento del vehículo. De esta manera, la figura 7, muestra los esfuerzos soportados por los neumáticos y las variables angulares y los esfuerzos que influyen en la dinámica del mismo (ángulo de deslizamiento, fuerzas y momentos en dirección positiva).
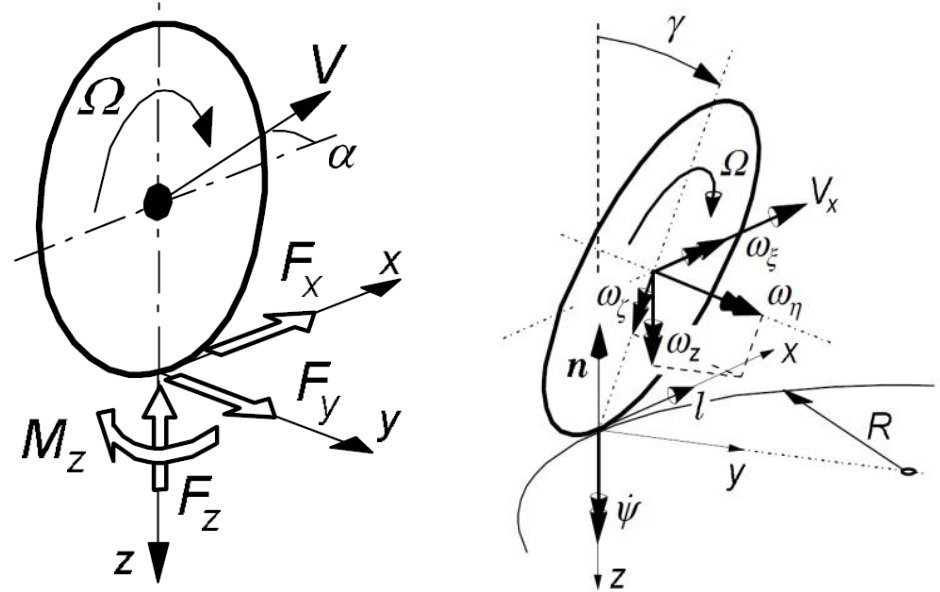

Fig 7. Factores característicos en los ejes de los neumáticos que pueden influir en las propiedades de manejo y estabilidad del vehículo. (Adaptada de Pacejka y Bakker, 1992)

La fórmula mágica se detalla a continuación en la ecuación 1,2 y 3

$$
\begin{aligned}
& y(x)=D \sin \left(C \tan ^{-1}\left\{B x-E\left[B x-\tan ^{-1}(B x)\right]\right\}\right) \\
& Y(X)=y(x)+S v \\
& x=X+S h
\end{aligned}
$$

Balkwill (2017) menciona que, la fórmula mágica genera una curva antisimétrica que pasa por el origen, alcanza un valor máximo y tiende a ajustarse a su asíntota horizontal como se muestra en la Fig 7 . El valor $y(x)$ es introducido de acuerdo con el eje del movimiento que se desea encontrar o variable de salida: eje y ( $F_{y}=$ Fuerza lateral), eje $z\left(M_{z}=\right.$ Momento Autoalineante), Eje $x\left(\mathrm{M}_{\mathrm{x}}=\right.$ Momento longitudinal). Los coeficientes de $\mathrm{B}, \mathrm{C}, \mathrm{D}$ y $\mathrm{E}$ corresponden a valores constantes característicos del neumático, como el coeficiente de rigidez o de amortiguación, e infieren en la forma de la curva obtenida, $X$ corresponde a la variable de entrada (ángulo de deslizamiento lateral o ángulo de deslizamiento longitudinal o el ángulo de deriva). Sv es el cambio vertical y Sh el cambio horizontal, estos desplazamientos tomando en cuenta los deslizamientos de rodadura y el cámber. Los coeficientes respecto a la carga nominal vienen dados por la tabla 3, los valores intermedios se deben interpolar.

Tabla 3: Valores de los coeficientes para B, C, D, E, Sh y Sv (Adaptada de García de Jalón, 2007)

\begin{tabular}{|l|l|l|l|l|l|l|l|l|}
\hline & Carga $(k N)$ & $B$ & $C$ & $D$ & $E$ & $S h$ & $S v$ & $B C D$ \\
\hline \multirow{4}{*}{ Fy } & 2 & 0.244 & 1.5 & 1936 & 0.132 & -0.28 & -118 & 780.6 \\
\cline { 2 - 9 } & 4 & 0.239 & 1.19 & 3650 & -0.678 & -0.049 & -156 & 1038 \\
\cline { 2 - 9 } & 6 & 0.164 & 1.27 & 5237 & -1.610 & -0.126 & -181 & 1091 \\
\hline \multirow{4}{*}{ Mz } & 2 & 0.247 & 2.56 & -15.53 & -3.920 & -0.464 & -12.5 & -9.82 \\
\cline { 2 - 9 } & 4 & 0.234 & 2.68 & -48.56 & -0.460 & -0.082 & -11.7 & -30.45 \\
\cline { 2 - 9 } & 6 & 0.164 & 2.46 & -112.5 & -2.040 & -0.125 & -6.00 & -45.39 \\
\hline \multirow{4}{*}{ Fx } & 2 & 0.178 & 1.55 & 2193 & 0.432 & 0 & 25 & 605 \\
\cline { 2 - 9 } & 4 & 0.171 & 1.69 & 4236 & 0.619 & 0 & 70.6 & 1224 \\
\cline { 2 - 9 } & 6 & 0.21 & 1.67 & 6090 & 0.686 & 0 & 80.1 & 2136 \\
\hline
\end{tabular}



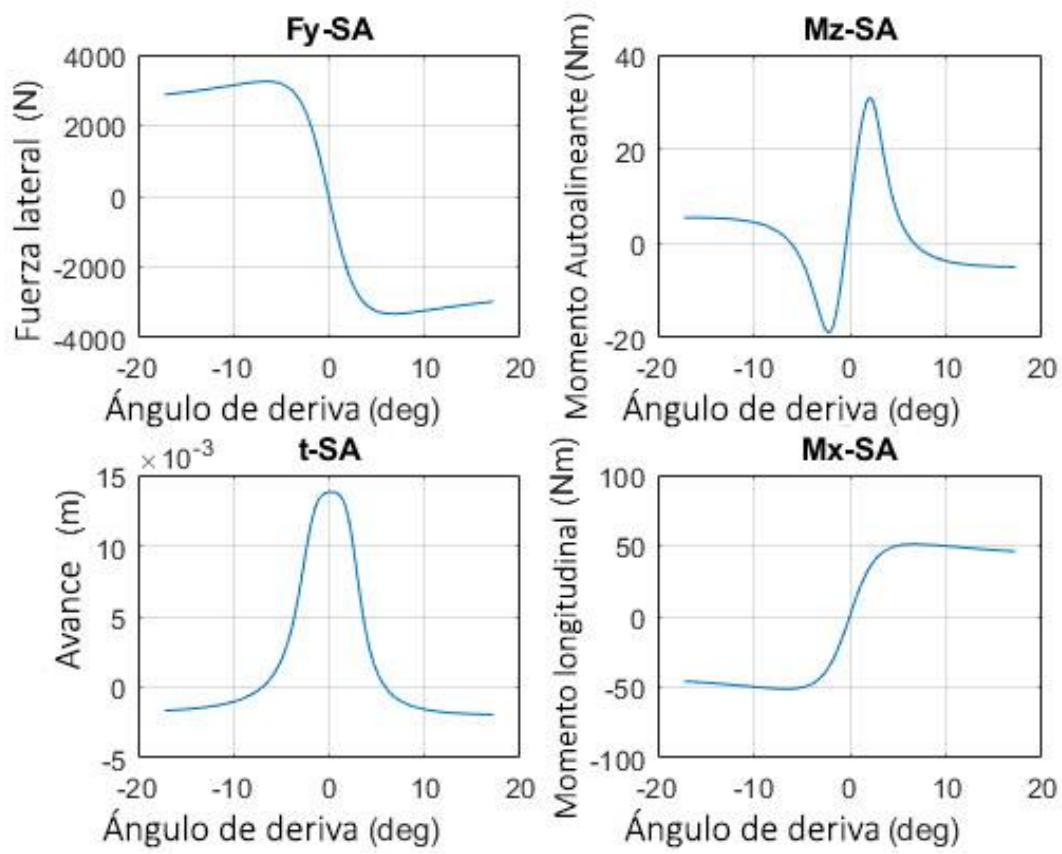

Fig 8. Valores resultantes producidos por la fórmula mágica de Pacejka (Adaptada de Mfval 2019)

\section{Método multicuerpo: Fórmulas del modelo de NTV}

El análisis de sistemas multicuerpo es una herramienta que permite modelar sistemas mecánicos como un conjunto de elementos sólidos rígidos y elementos flexibles, permitiendo obtener ecuaciones que determinan su comportamiento cinemático y dinámico mediante el estudio de sus condiciones de entorno, según figuras 9, 10 y 11. Estas ecuaciones dependen de los componentes del sistema, de su movimiento, de su deformación y se describen utilizando coordenadas que definen sistemas de referencia móviles. Furuichi et al., (2013) en donde, los sistemas de coordenadas están planteados de la siguiente manera:

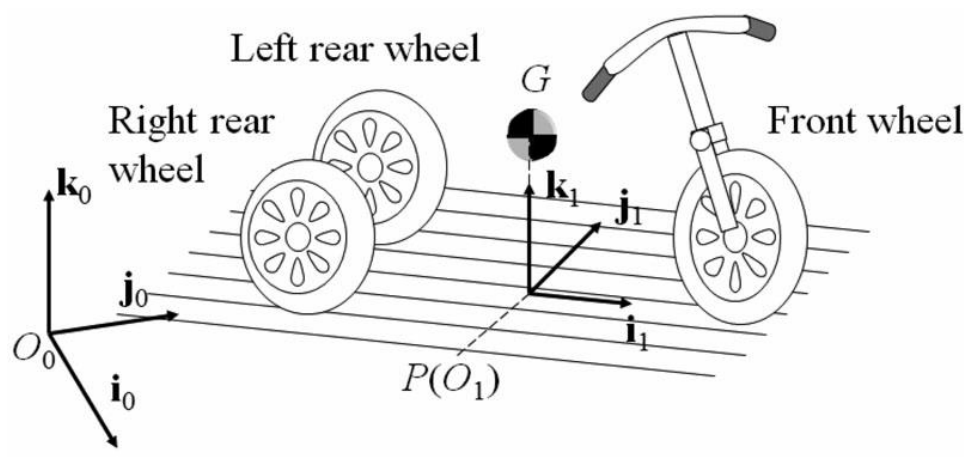

(a)
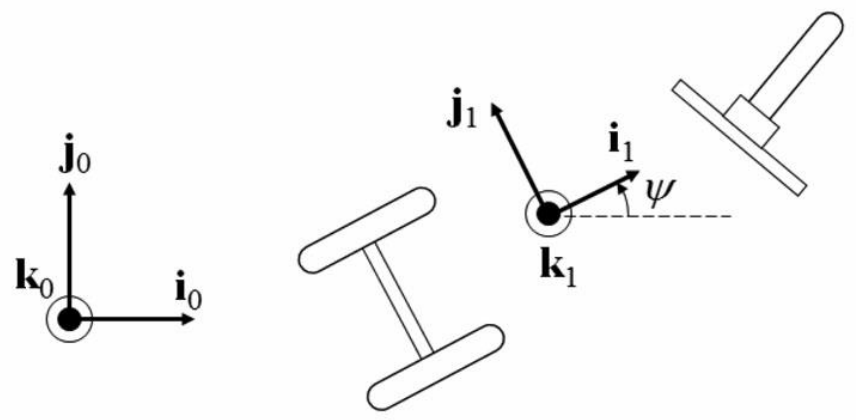

(b)

Fig 9. Sistemas de coordenadas $\{0\}$ y $\{1\}$. (a) Vista completa, (b) Vista superior (Adaptada de Furuichi et al., 2013) 


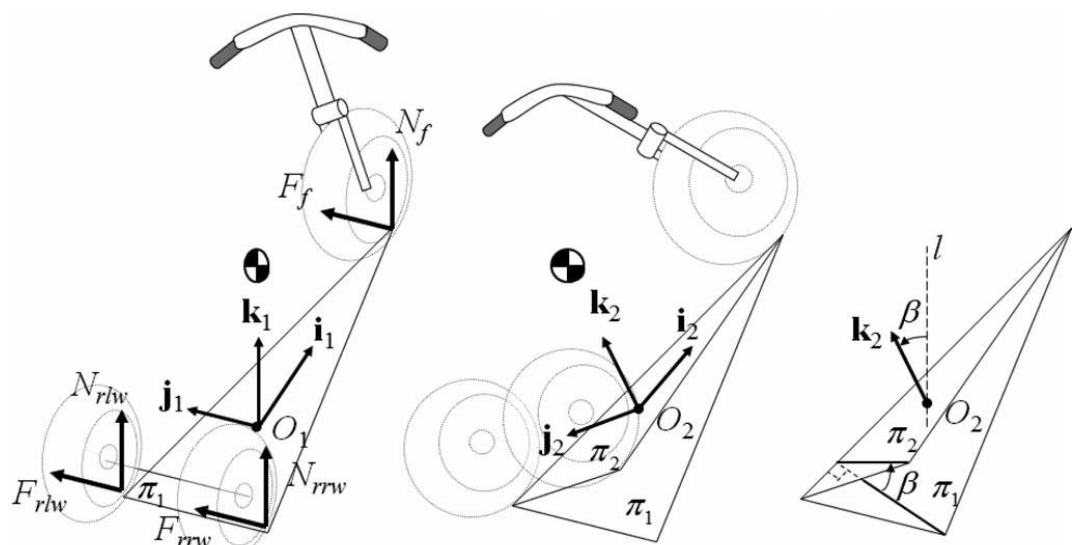

Fig 10. Sistemas de coordenadas $\{1\}$ y $\{2\}$. Vista completa del NTV con la rueda derecha levantada. (Adaptada de Furuichi et al., 2013)

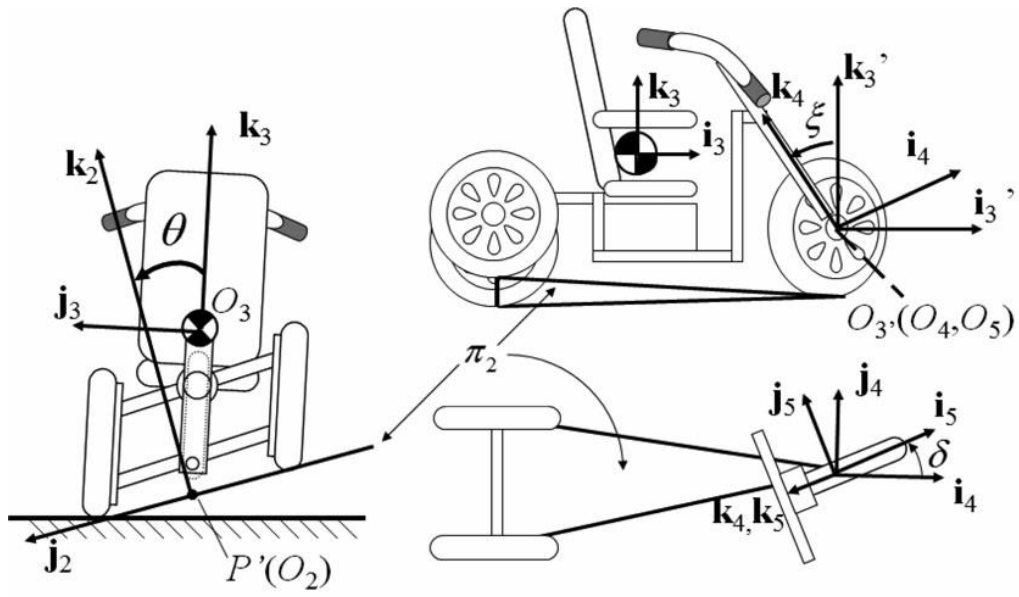

(a)

(b)

Fig 11. Sistemas de coordenadas (a) vista trasera, (b) vista lateral y superior (Adaptada de Furuichi et al., 2013)

Según Furuichi et al., (2013), las ecuaciones de movimiento lateral de traslación $y$, movimiento de vuelco $\beta$, movimiento de giro $\psi$, y movimiento de inclinación $\theta$ pueden obtenerse según las ecuaciones $4,5,6$ y 7 , estas ecuaciones representan el comportamiento dinámico del modelo NTV, cuyas variables están definidas en Furuichi et at., (2012).

$$
\begin{aligned}
& \begin{aligned}
& C_{\gamma} \cdot \ddot{y}=(\cdot)_{1} \cdot S_{\gamma} C_{\beta}+(\cdot)_{2} \cdot C_{\gamma} C_{\beta}+(\cdot)_{3} \cdot S_{\beta}-\ddot{\beta}\left(n_{\beta 1} \cdot S_{\gamma} C_{\beta}+n_{\beta 2} \cdot C_{\gamma} C_{\beta}+n_{\beta 3} \cdot S_{\beta}\right) \\
&-\ddot{\psi}\left(n_{\beta 1} \cdot S_{\gamma} C_{\beta}+n_{\psi 2} \cdot C_{\gamma} C_{\beta}+n_{\psi 3} \cdot S_{\beta}\right)-\ddot{\theta}\left(n_{\theta 1} \cdot S_{\gamma} C_{\beta}+n_{\theta 2} C_{\gamma} C_{\beta}+n_{\theta 3} \cdot S_{\beta}\right) \\
& \begin{aligned}
\left\{-n_{\beta 1} \cdot m h S_{\gamma} C_{\theta}\right. & \left.-n_{\beta 2} \cdot m h C_{\gamma} C_{\theta}-n_{\beta 3} \cdot m\left(l_{f} S_{\gamma}-h C_{\gamma} S_{0}\right)+e_{\beta 1}\right\} \ddot{\beta} \\
& =-(\cdot)_{1} \cdot m h S_{\gamma} C_{\theta}-(\cdot)_{2} \cdot m h C_{\gamma} C_{\theta}-(\cdot)_{3} \cdot m\left(l_{f} S_{\gamma}-h C_{\gamma} S_{\theta}\right)+(\cdot)_{4} \\
& -\ddot{y}\left\{-n_{y} \cdot m h S_{\gamma} C_{\theta}-n_{y 2} \cdot m h C_{\gamma} C_{\theta}-n_{y 3} \cdot m\left(l_{f} S_{y}-h C_{y} S_{\theta}\right)\right\} \\
& -\ddot{\psi}\left\{-n_{\psi 1} \cdot m h S_{\gamma} C_{\theta}-n_{\psi 2} \cdot m h C_{\gamma} C_{\theta}-n_{\psi 3} \cdot m\left(l_{f} S_{\gamma}-h C_{y} S_{\theta}\right)+e_{\psi 1}\right\} \\
& -\ddot{\theta}\left\{-n_{\theta 1} \cdot m h S_{\gamma} C_{\theta}-n_{\theta 2} \cdot m h C_{y} C_{\theta}-n_{\theta 3} \cdot m\left(l_{f} S_{\gamma}-h C_{\gamma} S_{\theta}\right)+e_{\theta 1}\right\}
\end{aligned} \\
&\left(e_{\psi 2} \cdot S_{\beta}-e_{\psi 3} \cdot C_{\beta}\right) \cdot \ddot{\psi}=(\cdot)_{5} \cdot S_{\beta}-(\cdot)_{6} \cdot C_{\beta}-\ddot{\beta}\left(e_{\beta 2} \cdot S_{\beta}-e_{\beta 3} \cdot C_{\beta}\right)-\ddot{\theta}\left(e_{\theta 2} \cdot S_{\beta}-e_{\theta 3} \cdot C_{\beta}\right) \\
&\left\{\left(l_{f} S_{\gamma} C_{\beta}-h C_{\gamma} C_{\beta} S_{\theta}-h S_{\beta} C_{\theta}\right) n_{\theta 3}-\frac{1}{m} C_{\beta} \cdot e_{\theta 1}\right\} \ddot{\theta} \\
&=\left(l_{f} S_{\gamma} C_{\beta}-h C_{\gamma} C_{\beta} S_{\theta}-h S_{\beta} C_{\theta}\right)(\cdot)_{3}-\frac{1}{m} C_{\beta}(\cdot)_{4}+\frac{1}{m} C_{\beta} C_{\gamma} M_{t} \\
&-\left(l_{f} S_{\gamma} C_{\beta}-h C_{\gamma} C_{\beta} S_{\theta}-h S_{\beta} C_{\theta}\right) n_{y 3} \ddot{y} \\
&+\left\{\frac{1}{m} C_{\beta} \cdot e_{\beta 1}-\left(l_{f} S_{\gamma} C_{\beta}-h C_{\gamma} C_{B} S_{\theta}-h S_{\beta} C_{\theta}\right) n_{\beta 3}\right\} \ddot{\beta} \\
&+\left\{\frac{1}{m} C_{\beta} \cdot e_{\psi 1}-\left(l_{f} S_{\gamma} C_{\beta}-h C_{\gamma} C_{B} S_{\theta}-h S_{\beta} C_{\theta}\right) n_{\psi 3}\right\} \ddot{\psi}
\end{aligned}
\end{aligned}
$$




\section{Sistema de control}

El éxito en el diseño del sistema de control es un reto, debido a que, el vehículo conjuga dos plataformas o sistemas de dirección. Por un lado, el ángulo de deriva depende del giro del timón y el giro de la rueda (automóvil), y por otro lado, el ángulo de deriva, también es afectado por la inclinación o caída que se le dé a las ruedas (motocicleta). De esta manera, el equilibrio entre el funcionamiento de los dos sistemas deberá proveer al NTV la seguridad y confort requeridos. En este sentido, se han planteado dos mecanismos que permitan direccionar al vehículo, estos son: Control directo de inclinación (DTC) y control de giro por inclinación (STC), los cuales interviene durante las maniobras de giro, cambiando la dirección de las ruedas y su inclinación empleando diferentes actuadores, siendo estos, hidráulicos, o electromecánicos.

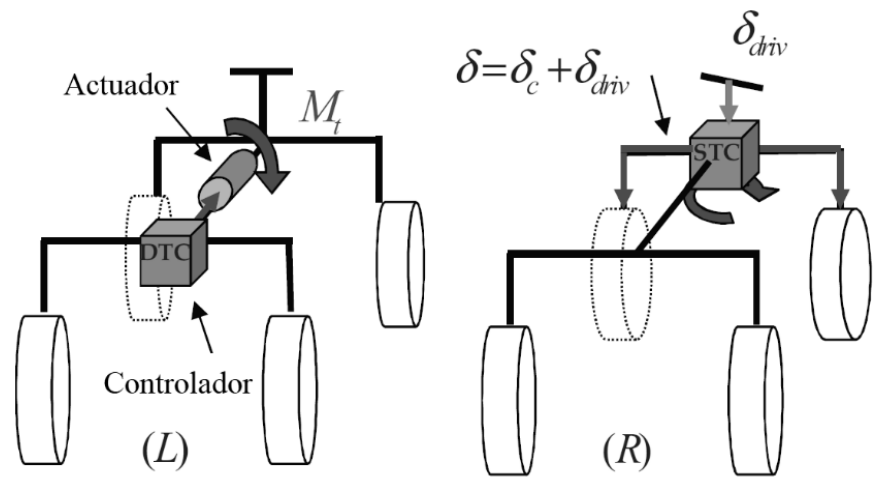

Fig 12. Sistema control DTC y STC (Adaptada de Claveau et al., 2014)

El sistema DTC está basado en un actuador dedicado como se indica en la figura 12 (L), montado en el eje longitudinal del NTV que provee el torque para inclinar el vehículo. Anteriormente los controladores para la suspensión activa inclinable eran diseñados calculando el ángulo deseado de inclinación dependiendo del giro de la dirección. Por su parte Dong et al., (2014) menciona que, el actuador STC requiere un sistema de dirección por cable (Steer by wire system) figura $12(\mathrm{R})$. El ángulo de dirección aplicado por el conductor es modulado por el sistema STC para controlar el ángulo de inclinación usando un contravolanteo. La estrategia de inclinación de esta manera se inspira en las acciones de control de una bicicleta o motocicleta, tomando como señal de entrada, la fuerza lateral al mismo tiempo.

Los sistemas STC son muy eficientes para las velocidades bajas, demandan un gran contravolanteo para inclinar al vehículo, lo que lo desvía significativamente de su trayectoria, al contrario del DTC puede ser más eficiente a alta velocidad. Según Robertson et al., (2014), para beneficiarse de las ventajas complementarias de ambos sistemas, algunos proyectos han involucrado el STC y el DTC para que trabajen a la par, y son mencionados como sistemas de control de giro - directo de inclinación (steering - direct tilt control o SDTC). El método de control directo de inclinación tiene un buen desempeño durante el estado estable o estacionario, pero las rápidas transcientes pueden conducir a un potencial vuelco, para investigar este fenómeno y diseñar un controlador mejorado se combina un modelo multicuerpo con un modelo dinámico de un vehículo de una sola pista para predecir el comportamiento de estado estable y transciente. Con este modelo, es posible obtener una representación precisa de la cinemática y dinámica del movimiento del vehículo. El modelo dinámico lateral simple, proporciona un fácil entendimiento y representación física del sistema, el cual, a menudo permanece oculto en un modelo multicuerpo complejo.

En la actualidad, las técnicas de control disponibles son muy numerosas. Junto a aquellas técnicas más tradicionales como el control por retroalimentación (PID), control mediante matriz dinámica (CMD) las cuales, según Chamorro et al., (2018) se caracterizan por su robustez y su bajo consumo de recursos computacionales e implementación. También, se han desarrollado muchas otras como las redes neuronales artificiales, los sistemas de lógica difusa o los algoritmos genéticos. Incluso estas técnicas, a su vez, admiten ser combinadas entre sí, por ejemplo, empleando una red neuronal para ajustar un control difuso.

\section{Control PID}

En su trabajo Claveau et al., (2014), manifiesta que algunas estrategias son utilizadas en el control de los NTVs, muchas usan estrategias basadas en el control una entrada, una salida, SISO (Single input Single Output) como son los controladores PD y PID, potenciadas por criterios lineales cuadráticos LQ, pero asumiendo un desacoplamiento entre la dinámica del vuelco y las otras magnitudes (giro, velocidad longitudinal, etc.). Por este motivo Utilizan en su prototipo controladores multivarialble para controlar la dinámica lateral del SDTC (STC+DTC) diseñados por medio de una teoría de control H2. Implementando también un control de ganancia programada para hacer el control más robusto, considerando de esta manera la dinámica longitudinal y la lateral en la dinámica del vehículo. 


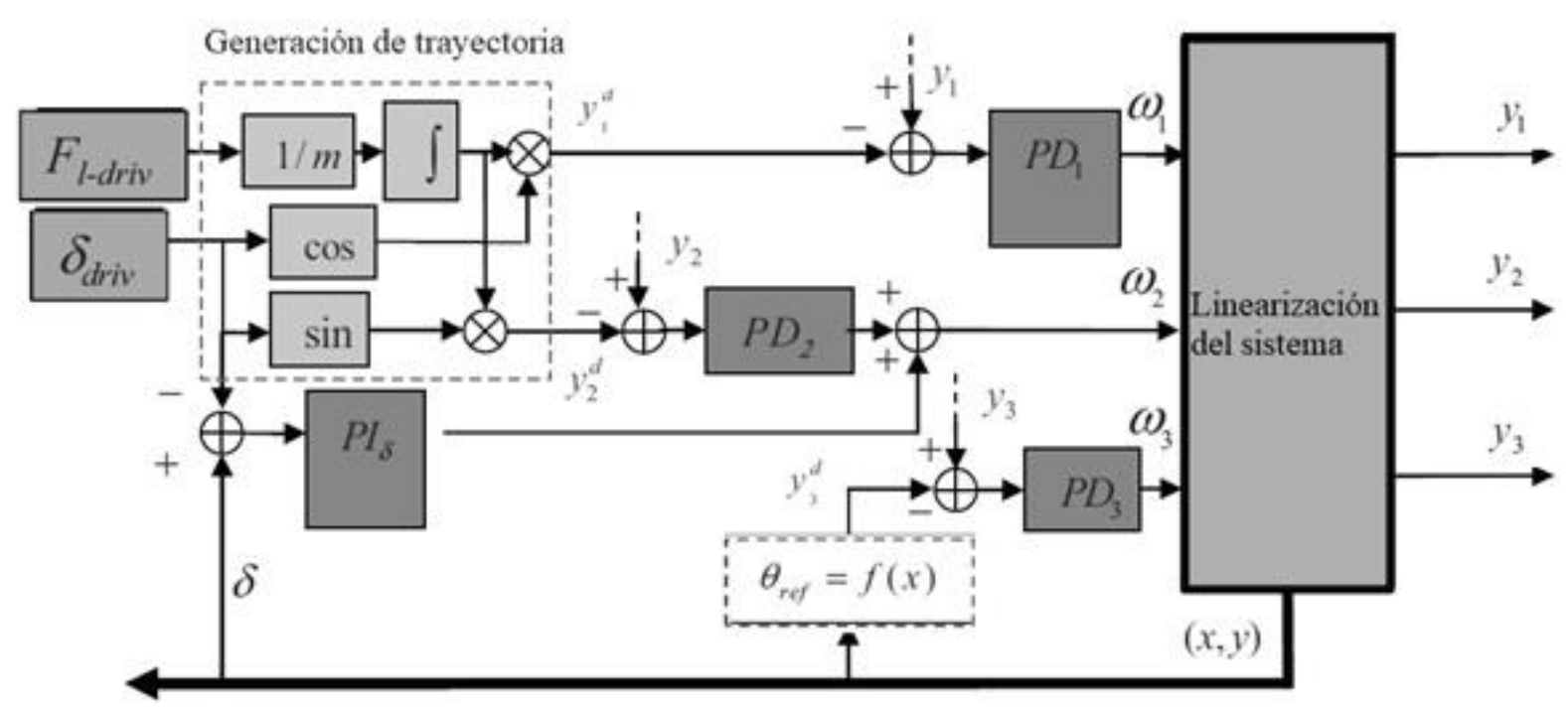

Fig 13. Control PID (Adaptada de Claveau et al., 2014)

El sistema de control implementado por Kim et al., (2014), hace referencia a un controlador PID, en donde la entrada es la relación de inclinación del vehículo para las entradas de la velocidad longitudinal y el ángulo de deriva ingresado por el conductor. Las constantes del controlador proporcional, integral y derivativo fueron ajustadas mediante la realización de varias simulaciones. Para el desarrollo del sistema de control se utilizan técnicas conocidas como son los controladores PID (Proporcional Integral Derivativo), figura 13, el cual es ampliamente aplicado en sistemas de control industrial, se trata de un sistema de control en lazo cerrado que calcula el error entre una magnitud medida y un valor deseado. El valor Proporcional depende del error actual. El Integral depende de los errores pasados y el Derivativo es una predicción de los errores futuros. La suma de estas tres acciones es usada para ajustar al proceso por medio de un elemento de control.

\section{Control CMD}

La tecnología de control por matriz dinámica (CMD) se basa en el principio de superposición, si se conoce la respuesta del sistema frente a cambios en la variable de entrada o frente a una perturbación medible, y se conoce cuál es el comportamiento de la variable del proceso, es posible encontrar la señal del controlador que mantenga al sistema funcionando dentro de los márgenes de funcionamiento. La estrategia de matriz dinámica presenta un mejor comportamiento que el control clásico (PID), teniendo menores desviaciones en las variables de control, proponiendo la implementación de esta estrategia avanzada como una alternativa atractiva debido a que permite desarrollar el control de varios lazos con la implementación de un solo sistema. Según Sánchez et al., (2015), el inconveniente que presentan los controladores por matriz dinámica, es su respuesta agresiva que, aunque hace que el sistema reaccione más rápido, provoca muchas oscilaciones, lo que puede causar que un proceso se torne inestable.

\section{Lógica difusa (Fuzzy Logic)}

Según Santos (2011), es comúnmente aceptado el hecho de que, en el control de sistemas complejos, hay requerimientos que hoy en día no pueden alcanzarse exclusivamente con la teoría del control convencional. Esto es debido, fundamentalmente, a la presencia de la incertidumbre que envuelve a los modelos mal definidos por falta de información, o a que la descripción analítica de esos sistemas es inabordable o conlleva excesiva complejidad computacional. El tratamiento de estos sistemas complejos o con un entorno de incertidumbre, en general no formulables en un riguroso marco matemático, ha conducido al desarrollo de los Controladores Inteligentes.

La lógica "fuzzy" o borrosa, es una ampliación de la lógica Boolena en donde una imprecisión inherente al lenguaje natural se representa de forma matemática. La teoría de conjuntos borrosos resulta útil en circunstancias en que los datos y sus relaciones no pueden escribirse en términos matemáticos precisos. Los conjuntos borrosos son una generalización de la lógica clásica y contienen objetos establecidos entre un rango de valores. El grado de pertenencia viene definido por una función de pertenencia, que usualmente toma valores entre 0 y 1 o verdaderos y falsos. El modelo mostrado en la figura 14, fue implementado por Zabala et al., (2018), y permite controlar un robot que balancea un péndulo invertido, el mismo que tiene un comportamiento muy parecido al implementado en las suspensiones inclinables, por esta razón se utiliza las leyes que rigen su movimiento para el control del NTV. 


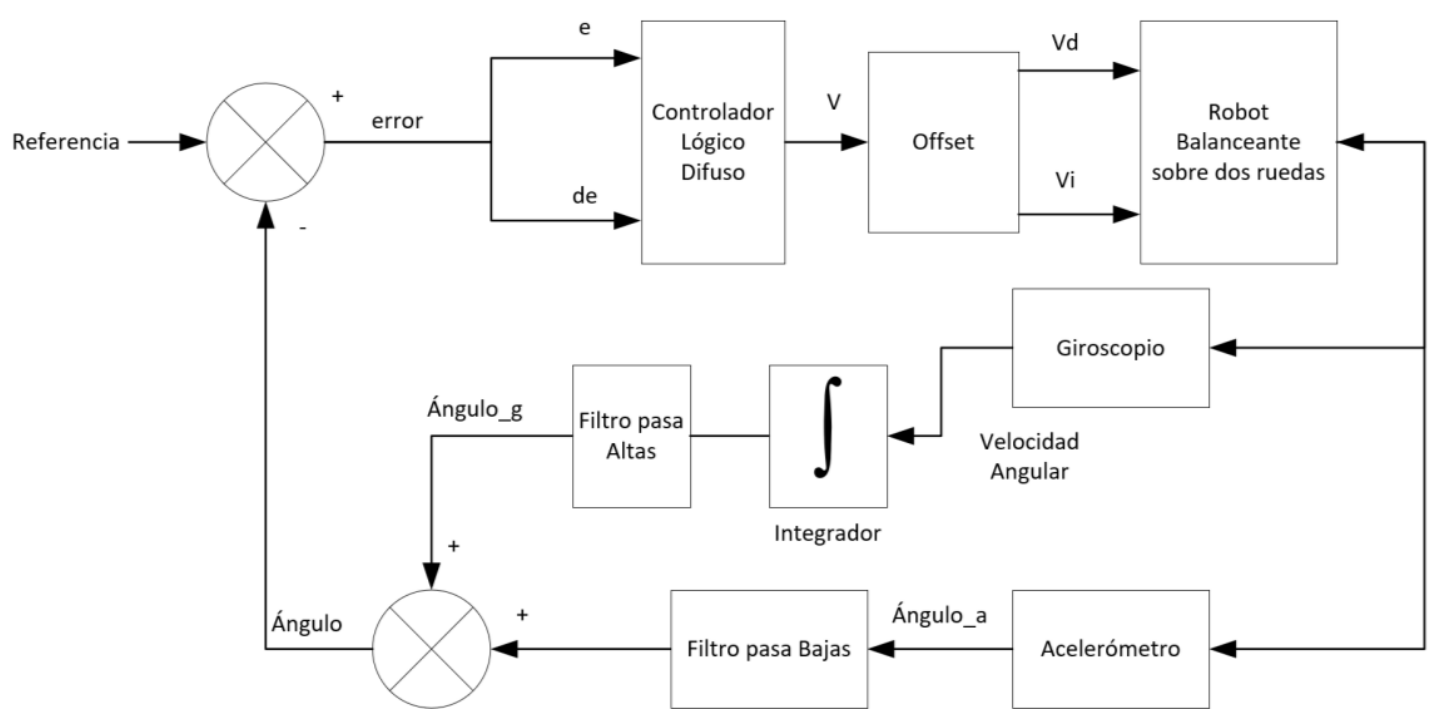

Fig 14. Diagrama de control combinado Fuzzy Logic - P, PID (Adaptada de Zavala et al., 2018)

\section{Redes Neuronales}

Son sistemas de control dinámicos, cuya estructura emula el proceso biológico del aprendizaje del ser humano. Se establece como red neuronal a un sistema compuesto por muchos elementos de procesamiento (neuronas) organizados en niveles o capas, cuya función es determinada por la estructura de la red, las conexiones y el procesamiento realizado por los elementos computacionales o nodos. En la figura 15 se muestra un sistema de red neuronal artificial propuesto por Durán et al., (2016) para el control del equilibrio de un péndulo invertido en donde se muestran sus componentes.

Entre las características de las redes neuronales se tiene que, pueden aprender de datos de entrenamiento, lo que llevaría a nuestro sistema a tener una adaptabilidad respecto a cada conductor, se ha demostrado que las redes neuronales pueden ser usadas con efectividad y precisión para la identificación y el control de sistemas con dinámicas complejas que resultan difícil de regular con métodos convencionales, esta función permitiría procesar señales de control inherentes a la seguridad activa y actuación del vehículo. En la vida real la mayoría de los procesos industriales pertenecen a esta categoría, de ahí la necesidad de métodos inteligentes para controlar esos sistemas. El interés creciente en las redes neuronales se debe a su gran versatilidad y al continuo avance en los algoritmos de entrenamiento de redes y en el hardware, lo que ha sido posible gracias a que cada vez es más fácil disponer de computadores extremadamente rápidos, a un precio competitivo, para implementar estos algoritmos (Santos, 2011).

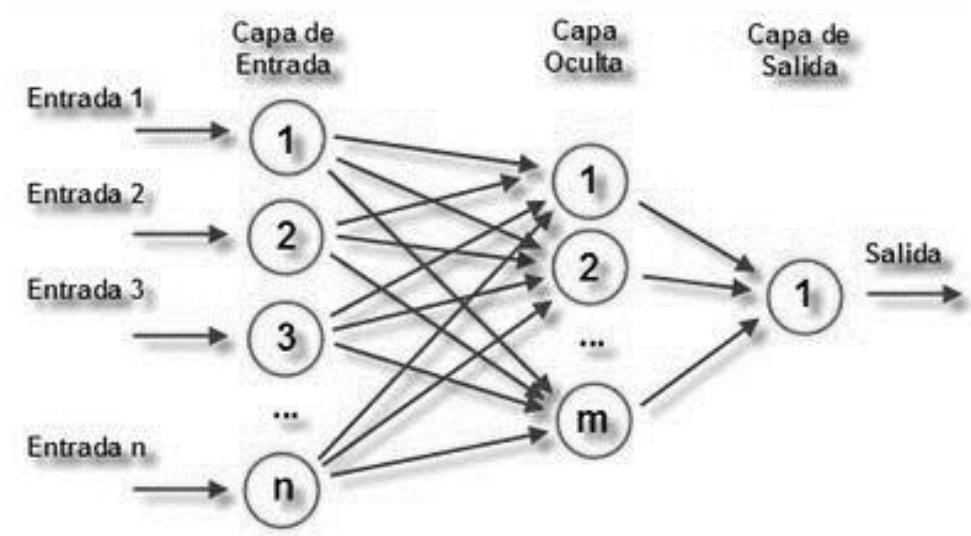

Fig 15. Estructura de una red neuronal artificial (Adaptada de De la Durán et al., 2016)

\section{Simulación}

La simulación computacional es una herramienta de diseño que permite reproducir sistemas mecánicos y de control, es decir componentes hardware y software de un sistema, máquina o proceso. Mediante la simulación se puede reproducir el comportamiento de los diferentes sistemas y componentes que conforman el vehículo y sus sistemas de control, esto se realiza con la finalidad de mejorar su desempeño con respecto al 
comportamiento de maniobrabilidad y confort. Las ventajas son inherentes al costo de diseño y producción, puesto que se valida primeramente el prototipo antes de su construcción. Los programas de simulación existentes permiten a los diseñadores determinar el comportamiento del vehículo con un alto grado de precisión, validando no solamente los componentes mecánicos además de sus sistemas de control.

\section{MATLAB - Simulink}

Arzola y Rojas (2013) en su trabajo evalúan una suspensión de paralelo deformable, el cual manifiesta una aproximación muy cercana al comportamiento deseado, el cual permite describir el comportamiento dinámico de un vehículo de cuatro ruedas, manteniendo una gran versatilidad y simplicidad, al momento de cambiar parámetros de entrada (dimensiones, rigidez amortiguación, etc.) obteniendo resultados sobre la dinámica longitudinal y transversal del vehículo bajo la acción de varias maniobras de conducción. En el área de diseño de nuevos dispositivos para el control del automóvil ha cambiado, actualmente se cuenta con asistencia computacional que permite diseñar sistemas de control en los vehículos que permiten mejorar el comportamiento dinámico, considerando algunas variables físicas del automóvil. El diseño de un sistema de control para la velocidad lateral es uno de los principales tópicos de investigación en el área de control de automóviles. Su propuesta se enfoca en el modelado matemático de un automóvil utilitario, validando su dinámica mediante el software simulink de Matlab y el entorno CarSim.

\section{MSC Adams}

Es un software de simulación de movimiento y prototipado, que permite modelar sistemas mecánicos y validar matemáticamente el movimiento tridimensional y las cargas estáticas y dinámicas para determinar el comportamiento del sistema bajo condiciones del mundo real. MSC Adams (Automatic Dynamic Analysis of Mechanical Systems) permite importar modelos gráficamente definidos, convertirlos en modelos dinámicos y validarlos mediante ecuaciones con sinnúmero de variables, dependientes de los grados de libertad, estas ecuaciones son analizadas mediante los principios de equilibrio cinemático y dinámico. Mejía et al., (2014) utilizan MSC Adams para la validación de un sistema de suspensión y manifiestan que esta metodología permite analizar sistemas de suspensión complejos basados en un número reducido de parámetros dinámicos sin la pérdida significativa de precisión, lo cual contribuye a la determinación de nuevos parámetros en las diferentes condiciones de operación.

Mitra et al., (2018) realiza la validación de los resultados en MSC Adams, para un sistema de suspensión implementando el modelo de un cuarto de vehículo, introduciendo como variables de entrada el cámber, el cáster, las masas suspendidas y no suspendidas, la presión en los neumáticos, la velocidad, coeficientes de rigidez y amortiguación. Los resultados del experimento arrojan similitudes entre sí, lo cual demuestra la aptitud del software para el desarrollo de este tipo de experimentos. La Figura 16 se presenta un banco de ensayos utilizado para la caracterización de los neumáticos.

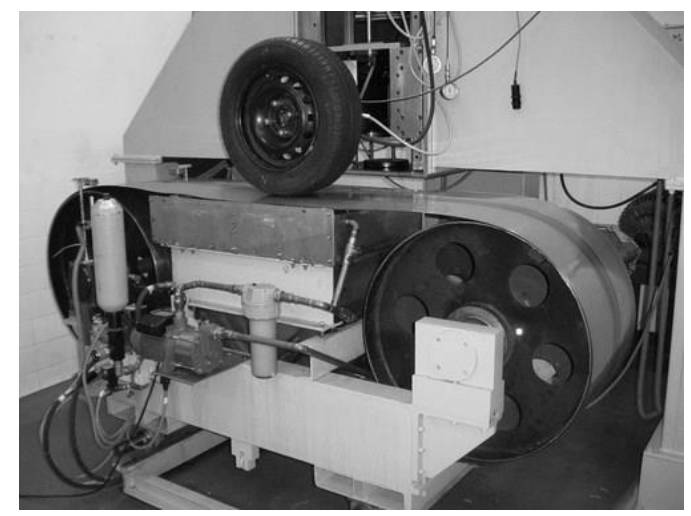

Fig 16. Experimento para validación de un cuarto de suspensión (Adaptada de Fernádez, 2019)

\section{SusProg}

Es una herramienta de diseño y análisis de sistemas de suspensiones vehiculares, posee varios modelos de suspensión que permiten al usuario realizar simulaciones y determinar los esfuerzos aplicados en cada uno de sus componentes. López-Monís (2018) en su proyecto emplea el software de simulación SusProg 3D con la finalidad de determinar el comportamiento dinámico de la suspensión en función de los diferentes ángulos de la geometría, en el cual se introducen diferentes valores para los ángulos comentados previamente. En resumen, la figura 17 muestra el proceso para el diseño y la validación de un sistema de suspensión inclinable para un vehículo angosto de transporte unipersonal. 


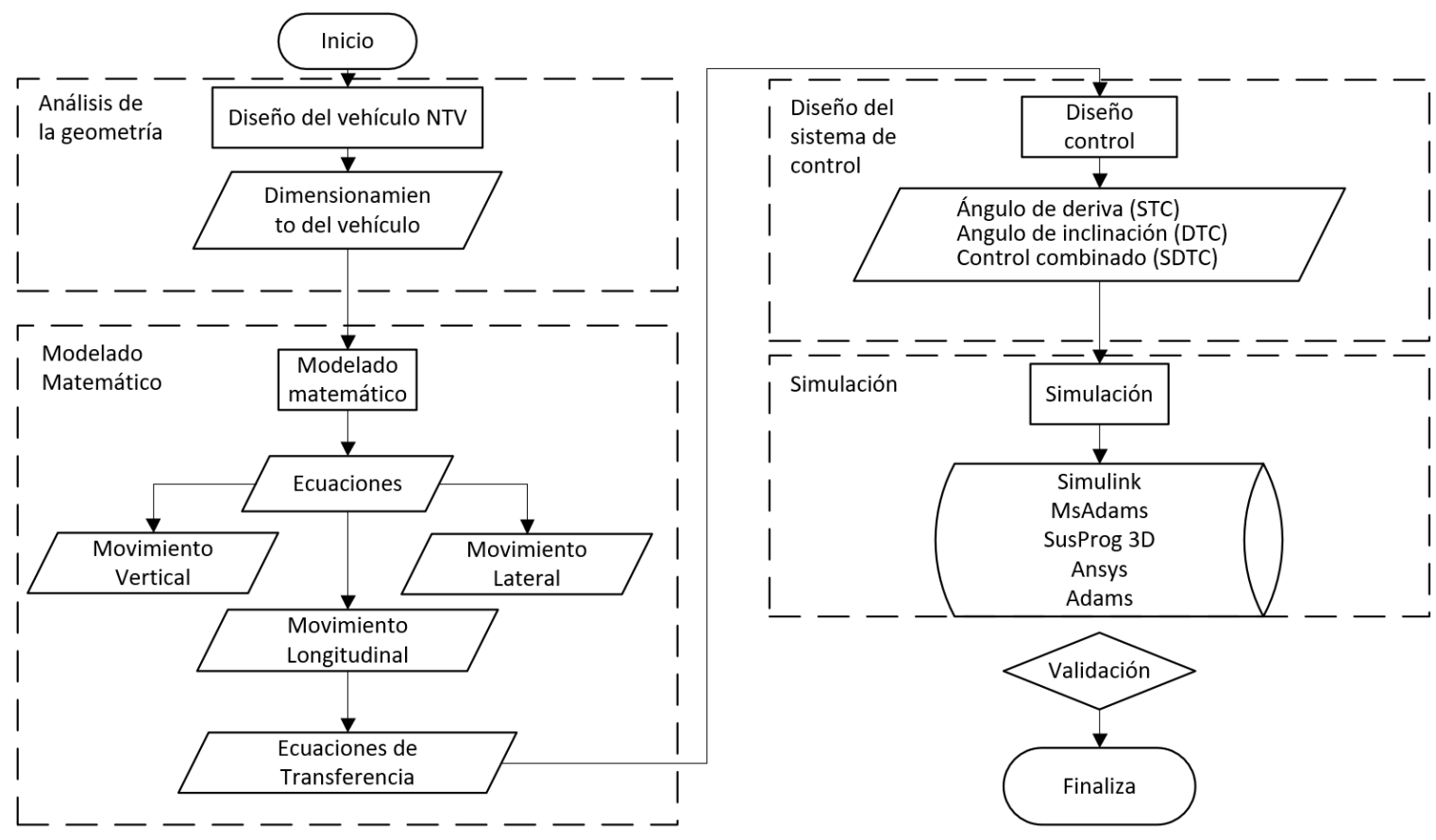

Fig 17. Esquema de la metodología

\section{DISCUSION FINAL}

El estudio de la suspensión tilting resulta importante para el desarrollo de un nuevo sector en el parque automotor, los vehículos unipersonales urbanos. El propósito es dar solución a los problemas de tránsito vehicular, existente en los sectores urbanos, consiguiendo mayor agilidad, eficiencia en el tránsito y disminución de la polución. Sin embargo, los factores sociales y económicos pueden ser un reto ante la adopción de esta nueva tecnología, de todas maneras, las estrategias de diseño, implementación de tecnologías actuales como: sistemas con control electrónico (frenos, dirección, transmisión, motor, etc.), motores híbridos o eléctricos, sistemas de seguridad activa y pasiva, unidos mediante sistemas de control de inteligencia artificial, podrían generar en los potenciales usuarios gran interés en cuanto a la adquisición de este tipo de transportación.

El resultado del análisis dinámico resulta de esta manera, una aproximación muy cercana a la realidad, siempre y cuando se realice tomando en cuenta todas las variables posibles para la ejecución del modelo. Además de la caracterización de cada uno de los componentes del sistema de suspensión. El método de control utilizado sigue siendo poco eficiente, puesto que debido a las diferentes formas de conducción es posible que no satisfagan las demandas de los usuarios, lo recomendable sería optar por tecnologías adaptativas e inteligencia artificial, que permitan al vehículo reconocer y adaptarse, tanto al usuario como a las condiciones de carretera presentes en tiempo real. En la actualidad los sistemas de simulación dedicados son herramientas fundamentales para el desarrollo de las nuevas tecnologías, permiten obtener aproximaciones significativas, pero debido a la infinidad de variables presentes en el comportamiento del vehículo resulta de vital importancia validar los datos de las simulaciones con prototipos reales. De esta manera se comprueba de manera exhaustiva y reiterativa los sistemas que proveen al vehículo de maniobrabilidad, estabilidad, seguridad y confort.

\section{CONCLUSIONES}

A partir del estudio de la literatura, se pueden mencionar los siguientes datos relevantes: 1) se establece las herramientas necesarias para la implementación del sistema de suspensión inclinable en los vehículos de transporte unipersonal, identificando primeramente la tipología de los vehículos unipersonales, particularmente los vehículos angostos inclinables NTV, sus dimensiones sistemas mecánicos, y de control y las estrategias de conducción; 2) se analiza el comportamiento dinámico lateral del vehículo, basados en la teoría de la Fórmula mágica de Pacejka y la teoría del péndulo invertido, con la finalidad de establecer las fórmulas que permitan predecir el comportamiento del vehículo al momento de realizar el giro; 3) por medio de métodos de linealización, se obtiene las ecuaciones de transferencia para la programación del controlador; 4) al evaluar los métodos tradicionales y programas de simulación se concluye que estos últimos ayudan a realizar la comprobación del modelo matemático; y 5) considerando las ventajas de los NTV, este tipo de transporte novedoso y sugerente merece un estudio más profundo, que permita desarrollar e implementar todos los sistemas tecnológicos posibles sobre ellos, más aún cuando se está migrando de lo convencional a lo tradicional. 


\section{REFERENCIAS}

Amodio, A., Corno M., Panzani, G., y Savaresi, S.M., Differential braking-based anti-rollover control for non-tilting narrowtrack vehicles, 2017 IEEE Conf. Control Tech. Appl. (CCTA) (2017).

Antony, J. J., y Jayabal, K., Rollover dynamics of a narrow tilting three-wheeled vehicle, In MATEC Web of Conferences, EDP Sci., 51, 01002 (2016).

Arzola, N., y Rojas, G., Análisis del comportamiento dinámico de un vehículo con suspensión independiente tipo paralelogramo deformable y barras de estabilidad transversal, Rev. Fac. de Ingeniería, 67, 112-125 (2013).

Balkwill, J., Performance Vehicle Dynamics: Engineering and Applications, Butterworth-Heinemann, (2017).

Berote, J., Darling, J., y Plummer, A., Lateral dynamics simulations of a three-wheeled tilting vehicle, Procee. Inst. Mech. Eng., Part D: J. of Automobile Eng., 229(3), 342-356 (2015).

Cardona, L.F., Faúndez, C., y Valderrama, J.O., Concepto de Similitud Geométrica para la Correlación de la Solubilidad Mutua en Mezclas Parcialmente Miscibles de Líquidos lónicos + Alcohol, https://doi.org/10.4067/S071807642019000300307, Inf. Tecnol., 30(3), 307-318 (2019).

Chamorro, W., y Rueda, P.V., Regulador cuadrático lineal y control predictivo aplicados en un sistema de cuatro tanques: una comparación de rendimiento, Enfoque UTE, 9(1), 204-216 (2018).

Chatterjee, M., Kale, M., y Chaudhari, B.N., Mathematical modelling of chassis dynamics of electric narrow tilting three wheeled vehicle, 2015 Annual IEEE India Conference INDICON, 1-6 (2015).

Claveau, F., Chevrel, P., y Mourad, L., Non-linear control of a narrow tilting vehicle, 2014 IEEE Int. Conf. Sys., Man, and Cybernetics (SMC), 2488-2494 (2014).

Corno, M., Panzani, G. y Savaresi, S.M., Single-track vehicle dynamics control: state of the art and perspective, IEEE/ASME Trans. Mechatronics, 20(4), 1521-1532 (2015).

de Dios, J. G., Álvarez, J. C. B., y Rodrigo, M. A., Listas guía de comprobación de revisiones sistemáticas y metaanálisis: declaración PRISMA, Evidencias en pediatría, 7(4), 20 (2011).

Dong, O., Graham, C., Grewal, A., Parrucci, C., y Ruina, A., The bricycle: a bicycle in zero gravity can be balanced or steered but not both, Vehicle Sys. Dynamics, 52(12), 1681-1694 (2014).

Durán, J. O. M., Herrera, N.C., Quintero, R.D.V., y Chaustre, Y.R., Control neuronal de un sistema de equilibrio (péndulo invertido) en dispositivos lógicos programables, Inv. e Innovación en Ing., 4(2), 40-51 (2016).

Furuichi, H., Huang, J., Fukuda, T., y Matsuno, T., Switching dynamic modeling and driving stability analysis of threewheeled narrow tilting vehicle. IEEE/ASME Transactions on Mechatronics, 19(4), 1309-1322 (2013).

Furuichi, H., Huang, J., Matsuno, T., y Fukuda, T., Dynamic model of three wheeled narrow tilting vehicle and corresponding experiment verification, IEEE/RSJ inter. conf. intelligent robots and systems, 3728-3733 (2012).

García de Jalón, J., Fórmula mágica de Pacejka, [ebook] Madrid: ETSII - Dep. Matemática Apli. Ing. Ind., pp.5,6. Available at: https://es.scribd.com/document/318469236/Magica-Formula [Accessed 26 Dec. 2018] (2007).

Guamán, E., Llanes-Cedeño, E. A., Celi-Ortega, S., y Rocha-Hoyos, J.C., Parámetros del Múltiple de Escape para su Diseño y Optimización Computacional: una revisión, http://doi.org/10.4067/S0718-07642019000600255, Inf. Tecnol., 30(3), 255-268 (2019).

Kim, S., McPhee, J., y Azad, N.L., Improving stability of a narrow track personal vehicle using an active tilting system, (No. 2014-01-0087), SAE Tech. Paper, (2014).

López-Monís Yuste, J. A., Estudio de la geometría de la suspensión frontal del Trophy Truck de Vildosola Racing, (2018). Ma, Z., Ji, X., Zhang, Y., y Yang, J., State estimation in roll dynamics for commercial vehicles, Veh. Sys. dynamics, 55(3), 313-337 (2017).

Maakaroun, S., Khalil, W., Gautier, M., y Chevrel, P., Modeling and simulating a narrow tilting car using robotics formalism, IEEE Trans. Intell. Transportation Sys., 15(3), 1026-1038 (2013).

de la Maza, L. E. L., de Cárdenas, L. Z., Ones, O. P., y Santiago, O. L., Obtención de un modelo neuronal para la estimación de la concentración de etanol en la destilería Héctor Molina, Tecnología Química, 38(2), 312-323 (2018).

Mejía, L. A., Valero,V., y Mata, V., Identificación de parámetros dinámicos de la suspensión de un vehículo, Ing. Competitividad, 16(1), 307-316 (2014).

Mitra, A. C., Fernandes, E., Nawpute, K., Sheth, S., Kadam, V., y Chikhale, S., Development and Validation of a Simulation Model of Automotive Suspension System using MSC-ADAMS, Mat. Today: Proc., 5(2), 4327-4334 (2018).

Mourad, L., Claveau, F., y Chevrel, P., Direct and steering tilt robust control of narrow vehicles, IEEE Trans. Int. Transportation Sys., 15(3), 1206-1215 (2014).

Nagarkar, M., Bhalerao, Y., Patil, G.V., y Patil, R.Z., Multi-Objective Optimization of Nonlinear Quarter Car Suspension System-PID and LQR Control, Proc. Manufacturing, 20, 420-427 (2018). 
Németh, B., y Gáspár, P., Nonlinear analysis and control of a variable-geometry suspension system, C. Eng. Prac., 61, 279-291 (2017).

Nguyen, A.-T., Chevrel, P., y Claveau, F., LPV Static Output Feedback for Constrained Direct Tilt Control of Narrow Tilting Vehicles, IEEE Trans. Control Sys. Tech. (2018).

Onsy, A. M., Sharaf, A.M., Ashrey, M. M., y Eldemerdash, S. M., Influence of Intelligent Active Suspension System Controller Design Techniques on Vehicle Braking Characteristics, SAE Inter. J. Veh. Dyn., Stability, and NVH, 3(10-03-010003) (2018).

Pacejka, H. B., y Bakker, E., The magic formula tyre model. Vehicle system dynamics, 21(S1), 1-18 (1992).

Robertson, J. W., Darling, J., y Plummer, A.R., Combined steering-direct tilt control for the enhancement of narrow tilting vehicle stability, Proc. Inst. Mech. Eng., Part D: J. Automobile Eng., 228(8), 847-862 (2014).

Rocha-Hoyos, J.C., Llanes-Cedeño, E. A., Celi-Ortega, S., y Peralta-Zurita, D., Efecto de la Mezcla de Biodiésel en el Rendimiento y la Opacidad del Motor Diésel, http://doi.org/10.4067/S0718-07642019000300137, Inf. Tecnol., 30(3), 137146 (2019).

Rocha-Hoyos, J.C., Tipanluisa, L., Zambrano, V., y Portilla, A., Estudio de un Motor a Gasolina en Condiciones de Altura con Mezclas de Aditivo Orgánico en el Combustible, http://doi.org/10.4067/S0718-07642018000500325, Inf. Tecnol., 29(5), 325-334 (2018).

Sánchez, E. J. I., García, J., Quintero, O., Calderón, S.M., y Acosta, A.R., Ecuaciones de Sintonización para Controlador por Modos Deslizantes y Control de Matriz Dinámica a partir de un Módulo Difuso, Rev. Cient. Axioma, 1(14), 14-24 (2015).

Santos, M., Un enfoque aplicado del control inteligente, Rev. Ibero. Automática e Informática Ind. RIAI, 8(4), 283-296 (2011).

Schommer, A., Soliman, P., Farias, L.T., y Martins, M., Analysis of a Formula SAE Vehicle Suspension: Chassis Tuning (No. 2015-36-0275), SAE Technical Paper (2015).

Smith, G., y Blundell, M., A new efficient free-rolling tyre-testing procedure for the parameterisation of vehicle dynamics tyre models. Proc. Inst. Mech. Eng., Part D: J. Automobile Eng., 231(10), 1435-1448 (2017).

Syed, U. H., y Vigliani, A., Vehicle side slip and roll angle estimation (0148-7191), (2016).

Tan, J. T. C., Arakawa, H., y Yoshihiro, S., Steering Dynamics of Tilting Narrow Track Vehicle with Passive Front Wheel Design, J. Physics: Conf. Series, IOP Publishing, 744(1), 012218 (2016).

Tognolli, A., Serafim, C.C.G., y Garcia, B.A., Study of Geometric Parameters for Validation and Reduction Effort in Steering System of a Vehicle FSAE (No. 2015-36-0147), SAE Technical Paper, (2015).

Valderrama, J. O., Principales Aspectos sobre la Preparación de un Artículo para ser Publicado en una Revista Internacional de Corriente Principal, http://doi.org/10.4067/S0718-07642005000200002, Inf. Tecnol., 16(2), 3-14 (2005)

Vega-William, H., Llanes-Cedeño, E.A., Molina, J., y Rocha-Hoyos, J.C., Revisión de las Características de Modelado y Optimización para el Diseño del Sistema de Suspensión Macpherson, http://doi.org/10.4067/S071807642018000600221, Inf. Tecnol., 29(6), 221-234 (2018).

Yao, J., Li, Z., Wang, M., Yao, F., y Tang, Z., Automobile active tilt control based on active suspension, Adv. Mech. Eng., 10(10), (2018).

Zavala, S. R., Morante, A.A.G., Villanueva, M.G., y García, V.T., Fuzzy-logic Control of a Two Wheeled Inverted Pendulum, XXXI Inter. Sum. Meet. power and Ind. Appli. (RVP-AI), 81-85 (2018). 